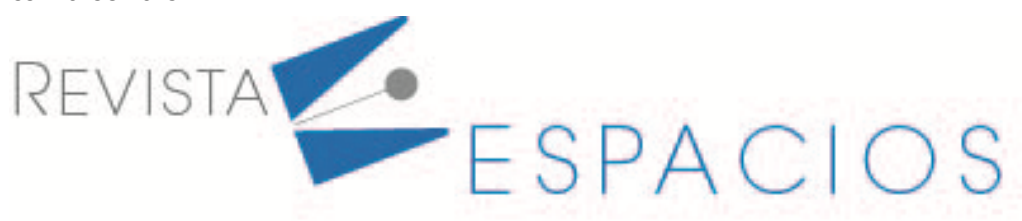

\title{
Caracterización mecánica de fibras nuevas y centenarias de agave americana $\mathrm{I}$.
}

\section{Mechanical characterization of new and centennial fibers of agave americana I.}

\author{
CAMPAÑA, Kleber O. ${ }^{1}$ \\ REMACHE, Abel P. ${ }^{2}$ \\ RE, María A. ${ }^{3}$
}

\begin{abstract}
Resumen
Se presentan los resultados de caracterizaciones mecánicas de tracción y tenacidad en fibras de agave americana I. nuevas y centarias. Las fibras nuevas fueron obtenidas por extracción manual y enriado, mientras que las fibras centenarias se obtuvieron manualmente de construcción antigua. Las fibras nuevas obtenidas por enriado presentaron mejores propiedades mecánicas, en comparación con las fibras nuevas y centenarias extraídas manualmente, mientras que las fibras centenarias vieron reducidas en un $38 \%$ sus propiedades mecánicas respecto a las obtenidas por enriado.

Palabras clave: agave americana l., fibras naturales, caracterización mecánica

Abstract

The results of mechanical characterizations of traction and tenacity in new and centennial agave americana I. fibers are presented.. New fibers were obtained by manual extraction and retting, while centennial fibers were obtained manually from an old construction. The new fibers obtained by retting showed better mechanical properties, compared to the new and centennial fibers extracted manually, while the centennial fibers had their mechanical properties reduced by $38 \%$ compared to those obtained by retting.
\end{abstract}

Keywords: agave americana I., natural fibers, mechanical characterization

\section{Introducción}

El agave americana (familia Asparagaceae, subfamilia Agavoideae, grupo Agaveae: ex Agavaceae s.s.) constituye un grupo de plantas monocotiledóneas que consta de 10 géneros y 340 especies cuya importancia en el desarrollo de las culturas mesoamericanas en zonas áridas es ampliamente reconocida. La larga relación entre los agaves y los seres humanos datan de hace 10,000 años, al comienzo de la agricultura en América. (De La Torre et al., 2018)

La hoja de agave americana se ha utilizado como fuente de fibra para la fabricación de cuerdas y telas gruesas, constituyéndose en un material importante en la antigua ingeniería andina. El procedimiento para su extracción incluye golpear o moler la hoja para extraer sus jugos y dejar la fibra desnuda. Ésta después se lava, eliminando

\footnotetext{
${ }^{1}$ Especialista en caracterización de materiales. Facultad de Ingeniería, Ciencias Físicas y Matemática . Universidad Central del Ecuador. kocampana@uce.edu.ec

${ }^{2}$ Profesor. Facultad de Ingeniería, Ciencias Físicas y Matemática. Universidad Central del Ecuador. apremache@uce.edu.ec

${ }^{3}$ Ingeniera Mecánica. Facultad de Ingeniería Mecánica . Universidad Internacional SEK. angelesre@uisek.edu.ec
} 
los restos de savia tóxica. Las fibras se enredan en cuerdas cada vez más grandes, para producir sogas ásperas pero muy resistentes. Las cuerdas de este tipo de fibra se usaban para amarrar las vigas procedentes de la misma planta en construcciones bio sustentables. (Bonifáz, 2018)

Aún quedan restos de casas construidas de esta manera, en pie tras más de cien años de exposición a los elementos ambientales. Este tipo de construcciones por utilizar materiales biodegradables y sostenibles, constituyen una potencial disminución de la contaminación e impacto ambiental que genera el sector de la construcción tradicional. La sociedad requiere considerar alternativas de bajo impacto ambiental para reemplazar materiales convencionales que se utilizan en los diferentes tipos de industrias. Una alternativa interesante es el uso de fibras naturales resistentes como el agave americana para aplicaciones estructurales.

Vale la pena señalar que aunque las fibras de agave americana tienen algunas características en común con otras fibras lignocelulósicas, sus propiedades aún no se han descrito completamente, considerando además que las propiedades de las fibras naturales dependen del origen, la edad y las técnicas de separación de las fibras de sus fuentes parentales (Thakur et al., 2013). Por lo tanto, para permitir su uso en la industria textil o en sector de la construcción se deben analizar los aspectos básicos de su estructura y morfología, como la composición química, la cristalinidad, la densidad y las propiedades mecánicas de tracción. (Bezazi et al., 2014; El Oudiani et al., 2009)

La resistencia mecánica es una propiedad importante de las fibras naturales, ya que puede variar de una fuente a otra y depende en gran medida de causas como factores nutricionales o climáticos que a menudo pueden estar fuera del control humano. Los conceptos de fuerza, alargamiento, elasticidad, resistencia a la abrasión y flexibilidad son importantes para evaluar la durabilidad de las fibras. Se requiere un buen conocimiento de estas propiedades de rendimiento para evaluar la durabilidad (Mafaesa, 2006).

La importancia de este estudio radica en contrastar las propiedades mecánicas evaluadas en fibras de agave americana I. centenarias con respecto a las evaluadas en fibras nuevas obtenidas por extracción manual y enriado, para determinar como afecta el envejecimiento natural en la resistencia a tracción y tenacidad de este tipo de fibras. De esta manera se puede discurrir en cuales aplicaciones sería adecuado el uso de estas fibras como refuerzo.

\section{Metodología}

En este trabajo de investigación se obtuvieron fibras nuevas de agave americana I. por los métodos de extracción manual y enriado con y sin cutícula, y también la extracción manual de fibras centenarias procedentes de una construcción andina antigua. Para evaluar la densidad lineal de las fibras se utilizó como referencia la norma ASTM D861-07, mientras que para evaluar las propiedades de esfuerzo a la ruptura y tenacidad se utilizó como referencia la norma ASTM D2256-10.

Se utilizaron tres grupos de fibras de agave americana I. Como se puede apreciar en la tabla 1, los grupos se diferencian entre sí por el método de extracción, proceso de secado y tiempo de existencia. Para la obtención de las fibras nuevas, se tomaron cinco hojas de una planta que se encontró en la periferia de la provincia de Pichincha (Ecuador), estas hojas tuvieron en promedio 1,90 $\mathrm{m}$ de largo y $0.17 \mathrm{~m}$ de ancho. Las hojas fueron sometidas a dos procesos para la extracción de la fibra. Unas secadas al ambiente, mientras que otras se sometieron a un proceso de enriado. 
Tabla 1

Grupos de fibras de agave americana L. preparadas

\begin{tabular}{cc}
\hline Identificación del grupo & Descripción \\
\hline AAL-M & Fibras de agave americana l. extraídas manualmente y secadas al ambiente \\
\hline AAL-E & Fibras de agave americana I. extraídas por enriado \\
\hline AAL-C & Fibras Agave americana Lineé de aproximadamente 100 años de antigüedad \\
\hline & Fuente: elaboración propia
\end{tabular}

\subsection{Fibras del grupo AAL-M}

Dos de las cinco hojas se secaron en una habitación cerrada, con luz solar a traves de una ventana, sin corriente de aire, a temperatura ambiente entre $22^{\circ} \mathrm{C}$ y $25^{\circ} \mathrm{C}$, durante 10 días. A una de las hojas se le retiró la cutícula (figura 1), con el fin de realizar posteriormente la comparación de las características con las de la hoja a la que no se le retiro la cutícula. Una vez que la hoja estuvo seca se realizó la liberación de la capa superficial sin cutícula, la cual se encontraba lista para tomar las muestras de fibra. Las fibras se limpiaron manualmente y se secaron al ambiente, para luego ser guardadas en un lugar sin humedad.

Figura 1

Proceso de extracción de la cutícula de la hoja de agave americana I.

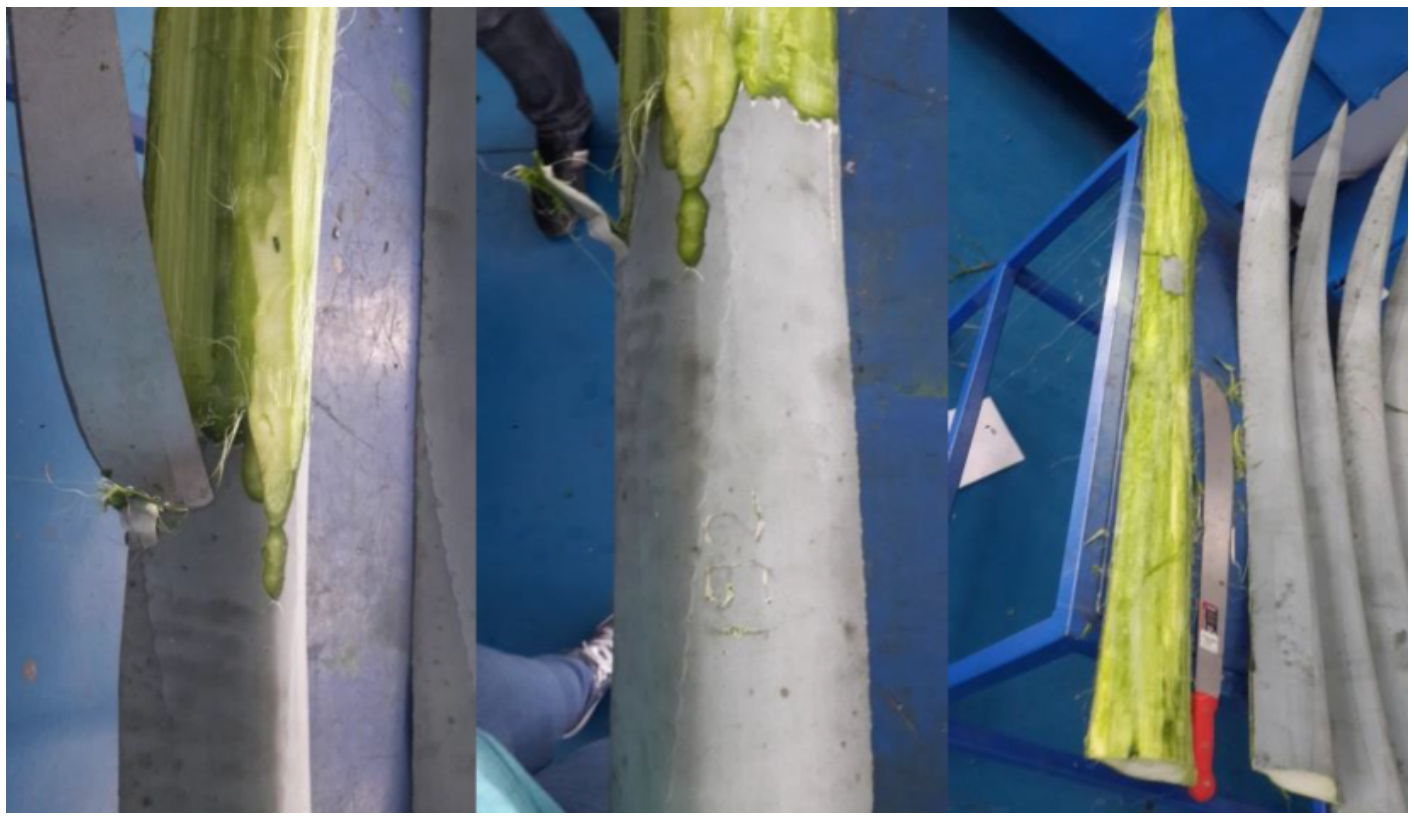

Fuente: elaboración propia

\subsection{Fibras del grupo AAL-E}

El proceso de enriado consistió en sumergir totalmente las hojas en agua, dentro de un recipiente cerrado con capacidad de 12 litros, por un periodo de 13 días. De manera análoga que en el proceso de secado al medio ambiente, se utilizó un fragmento de hoja con cutícula y otro sin cutícula. Para el décimo tercer día se procedió a retirar las hojas del tanque y se extrajeron las fibras (figura 2).

Las fibras obtenidas con este proceso deben ser lavadas unicamente con agua, para evitar cualquier cambio en las propiedades fisicas de las mismas y poder obtener resultados reales de la materia prima obtenida. Eliminando en esta etapa la parte blanda residual del enriado. Posteriormente las fibras fueron secadas durante un periodo de setenta y dos horas en un lugar totalmente cubierto, con corriente de aire, expuestas al sol y a temperatura 
ambiente. Finalmente, se procedió a la limpieza manual de cada una de las fibras, con el objeto de acondicionarlas previo a los ensayos de caracterización mecánica.

Figura 2

Extracción de la fibra de agave americana I. con el proceso de enriado

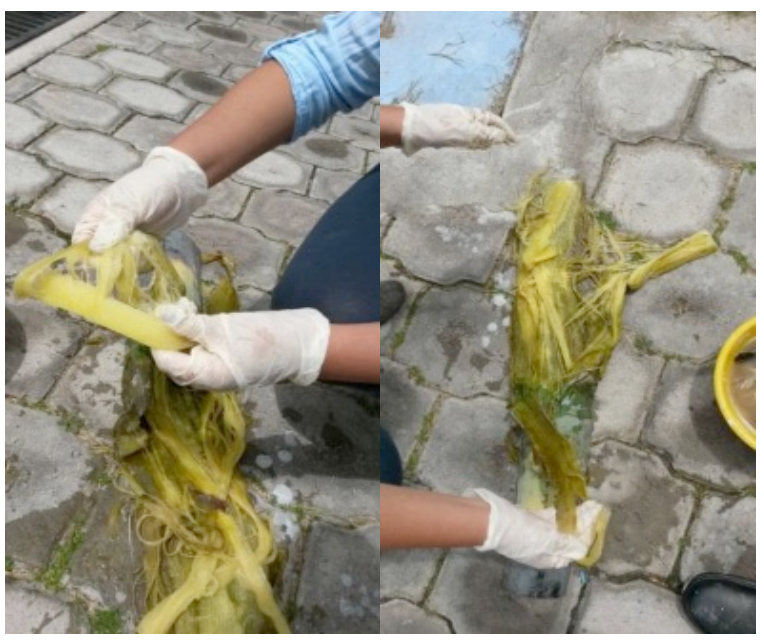

Fuente: elaboración propia

\subsection{Fibras del grupo AAL-C}

Estas fibras de agave americana I. fueron obtenidas de los elementos de unión de travesaños del techo de una vivienda rústica con más de 100 años de antigüedad, ubicada en el sector de Collaqui (Quito - Ecuador) (figura 3.). Las fibras se obtuvieron y limpiaron manualmente como acondicionamiento previo a su caracterización.

Figura 3

Vivienda rústica de cuyo techo se extrajeron las fibras de agave americana I. centenarias.

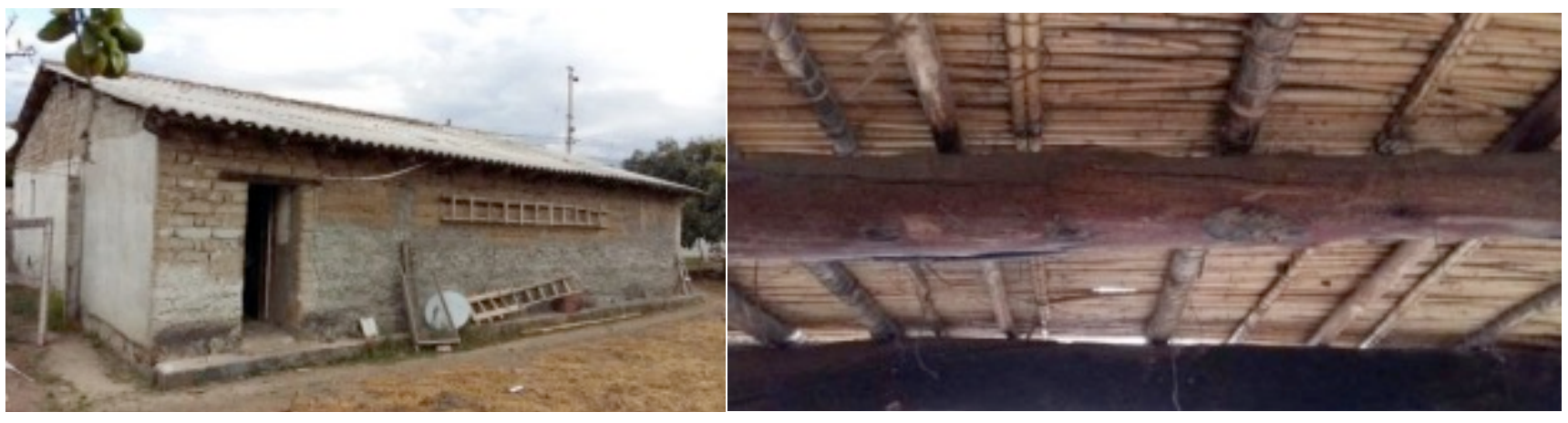

Fuente: elaboración propia

\subsection{Obtención del título de la fibra}

El título o densidad lineal de la fibra es un dato importante que se utiliza en el cálculo del diámetro de la fibra. A partir de este dato se puede obtener el área de la fibra, lo que a su vez se utiliza para calcular el esfuerzo a la ruptura, tomando en cuenta que éste es la razón entre la fuerza de ruptura y el área de la sección transversal de la fibra al inicio del ensayo. Para la obtención del título o número de la fibra se siguieron los lineamientos de la norma ASTM D861-07, para lo cual se midió la longitud de cada una de las fibras con un calibrador con pinzas en 
los extremos que mantienen las fibras extendidas durante el proceso de medición. Mientras que para obtener la masa de cada uno de los filamentos de fibra, se utilizó una balanza analítica marca KERN, modelo ABS220-4; con una apreciación de 0,1 mg.

\subsection{Caracterización mecánica de las fibras}

Los ensayos de tracción y tenacidad fueron realizados siguiendo los lineamientos de la norma ASTM D2256-10. Para esto se utilizó una máquina universal de ensayos marca Instron, modelo 3345; con una celda de carga de $500 \mathrm{~N}$ que permite una apreciación de 0,01 N. Las velocidades de ensayo se regularon para que las probetas se rompan en un tiempo de $20 \pm 3 \mathrm{~s}$.

\section{Resultados}

En la tabla 2 se reportan los valores de la resistencia a la ruptura de los tres grupos de fibras con su respectivo coeficiente de variación (CV). El promedio del esfuerzo a la ruptura de las fibras del grupo AAL-E fue mayor en un $24 \%$ y $38 \%$ comparado con los promedios del esfuerzo a la ruptura de las fibras de los grupos AAL-M y AAL-C respectivamente. Asimismo, se observa un coeficiente de variación relativamente alto, principalmente en el grupo de mayor resistencia a la ruptura AAL-E. De acuedo con Hulle et al. (2015) las propiedades de tracción de la fibra de agave americana no son uniformes. Esto se puede explicar por el hecho de que es una fibra natural y las fibras naturales están sujetas a irregularidades de crecimiento en la medida en que las fibras de la misma planta no son uniformes en tamaño y propiedades. Las vainas exteriores de las hojas producen las fibras más fuertes, mientras que las vainas interiores producen las fibras más débiles. Las fibras más internas tienen una alta resistencia a la tracción mientras que las fibras periféricas tienen menor resistencia a la tracción.

Tabla 2

Resultados de los ensayos a tracción de los tres grupos de fibras evaluados.

\begin{tabular}{ccc}
\hline Grupo & \multicolumn{2}{c}{ Esfuerzo a la ruptura } \\
\cline { 2 - 3 } Id. & {$[\mathrm{MPa}]$} & $\mathrm{CV}[\%]$ \\
\hline AAL-E & 449,70 & 30,26 \\
\hline AAL-M & 340,54 & 19,96 \\
\hline AAL-C & 278,24 & 18,03 \\
\hline & Fuente: elaboración propia
\end{tabular}

En la figura 4 se observan los comportamientos de la resistencia a la ruptura de los tres grupos de fibras ensayadas y se aprecia una superioridad marcada en esta propiedad del grupo AAL-E respecto a los grupos AAL$M$ y AAL-C, asimismo se aprecia una diferencia significativa de esta propiedad entre los grupos AAL-M y AAL-C, siendo la el promedio de resistencia a la ruptura del grupo AAL-M superior en un $18 \%$ respecto a esta propiedad en el grupo AAL-C. 
Figura 4

Comportamiento de la resistencia a la ruptura de los tres grupos de fibras analizadas.

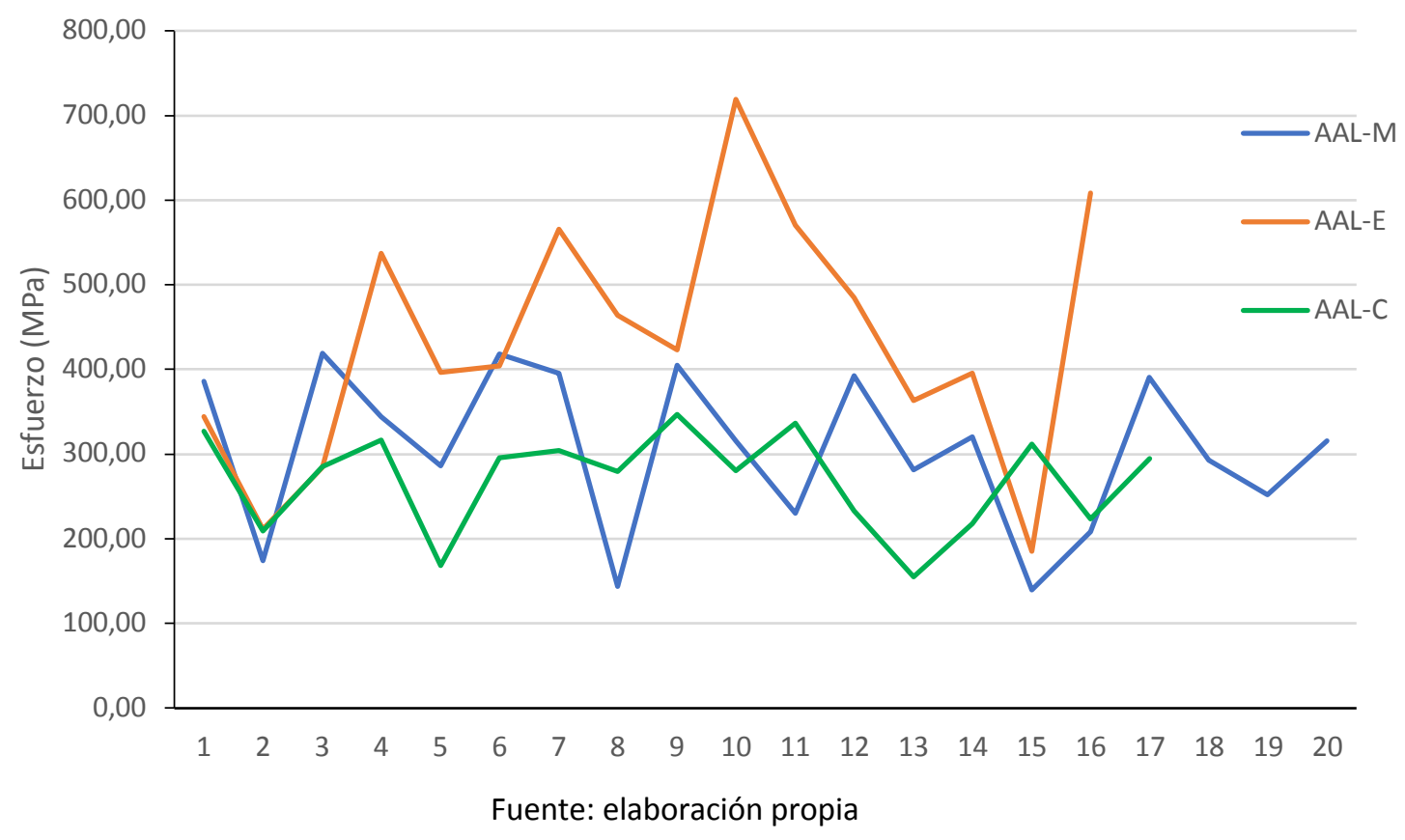

En la tabla 3 se reportan los valores promedios de tenacidad para los tres grupos de fibras. De forma similar que para la resistencia a la ruptura, se puede observar que las fibras del grupo AAL-E presentan el valor promedio mas alto de tenacidad, siendo superior en un $24 \%$ y $38 \%$ respecto a la tenacidad promedio de los grupos AAL-M y AAL-C respectivamente. Las mejores propiedades mecánicas se han evidenciado en el grupo AAL-E, cuyas muestras fueron obtenidas por enriado, esto concuerda con lo expuesto en el trabajo de Mafaesa (2006), argumentando que las fibras celulósicas naturales son las únicas fibras que aumentan sus propiedades mecánicas cuando están mojadas.

Resultados similares fueron reportados por Msahli et al. (2006) en el que las fibras de agave americana I. extraidas por enriado en agua de mar presentan una tenacidad promedio de $28,29 \mathrm{cN} /$ tex con un coeficiente de variación de $22,92 \%$.

\section{Tabla 3}

Resultados de los ensayos de tenacidad en los tres grupos de fibas evaluados.

\begin{tabular}{ccc}
\hline \multirow{2}{*}{$\begin{array}{c}\text { rrupo } \\
\text { Id. }\end{array}$} & \multicolumn{2}{c}{ Tenacidad } \\
\cline { 2 - 3 }$[\mathrm{cN} /$ tex $]$ & CV [\%] \\
\hline AAL-E & 32,1 & 30,2 \\
\hline AAL-M & 24,3 & 20,0 \\
\hline AAL-C & 19,9 & 18,0 \\
\hline \multicolumn{2}{c}{ Fuente: elaboración propia }
\end{tabular}

En la figura 5 se observan los comportamientos de la tenacidad de los tres grupos de fibras ensayadas y de igual forma que en los resultados de los ensayos de tracción para determinar la resistencia a la ruptura, se aprecia una superioridad en cuanto a la tenacidad del grupo AAL-E respecto a los grupos AAL-M y AAL-C, asimismo se aprecia una diferencia significativa de esta propiedad entre los grupos AAL-M y AAL-C, siendo la el promedio de tenacidad del grupo AAL-M superior en un $18 \%$ respecto a esta propiedad en el grupo AAL-C. 
Figura 5

Comportamiento de la tenacidad de

los tres grupos de fibras analizadas.

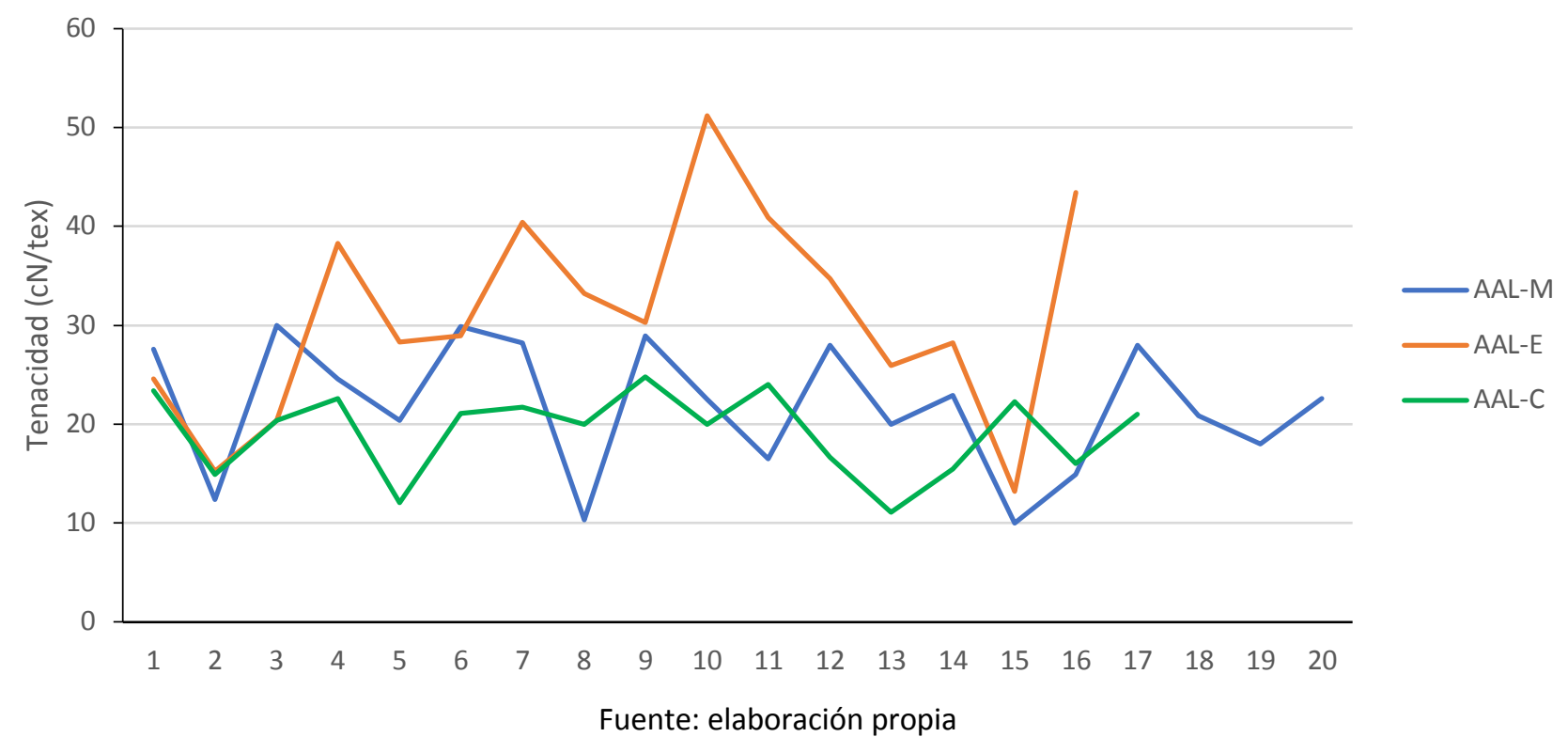

\section{Conclusiones}

Los resultados de los ensayos de resitencia a tracción y tenacidad evidenciaron que las fibras de agave americana I. obtenidas mediante el proceso de enriado presentan las mejores propiedades mecánicas, hasta en un $24 \%$ en relación con fibras obtenidas mediante extracción manual. Esto debido a que en el proceso de enriado las hojas de agave americana I. tuvieron una buena hidratación para extraer sus fibras.

Las fibras de agave americana I. centenaria vieron reducidas sus propiedades mecánicas en un 38\% en relación con las fibras nuevas obtenidas por enriado. El envejecimiento temporal de las fibras hace que éstas se resequen y se fragilicen, lo que constituiría un inconveniente al momento de utilizarlas en aplicaciones estructurales. Sin embargo, si las fibras fuesen incorporadas a una matriz que la proteja del medio ambiente, puede llegar a constituirse un material compuesto con potenciales aplicaciones en la industria textil, construcción y automotriz, debido a la baja densidad de las fibras y su menor impacto ambiental respecto a fibras sintéticas.

Se observó que las fibras obtenidas por enriado con cutícula y sin cutícula se degradaron de igual manera, en el mismo periodo de tiempo y los resultados de las pruebas mecánicas de tracción y tenacidad fueron muy similares. Por lo tanto se puede decir que es factible utilizar hojas con cutícula, lo cual evitaría el trabajo de la eliminación de la misma, reduciendo el tiempo del proceso.

\section{Referencias bibliográficas}

American Society for Testing and Materials. (2018). ASTM D861-07: Standard practice for use of the tex system to designate linear density of fibers, yarn intermediates, and yarns. https://doi.org/10.1520/D0861-07R18.

American Society for Testing and Materials. (2015). D2256/D2256M - 10: Standard test method for tensile properties of yarns by the single-strand method. https://doi.org/10.1520/D2256_D2256M-10R15.

Bonifáz, G. (13 de diciembre de 2018). El Ágave, Allpa. Recuperado de: https://www.allpa.org/el-agave/ 
Bezazi, A., Belaadi, A., Bourchak, M., Scarpa, F., \& Boba, K. (2014). Novel extraction techniques, chemical and mechanical characterisation of Agave americana L. natural fibres. Composites Part B: Engineering, 66, 194-203. https://doi.org/10.1016/j.compositesb.2014.05.014

De La Torre, L., Cummins, I., \& Logan-Hines, E. (2018). Agave americana and Furcraea andina: Key Species to Andean Cultures in Ecuador. Botanical Sciences, 96(2), 246-266. https://doi.org/10.17129/botsci.1813

El Oudiani, A., Chaabouni, Y., Msahli, S., \& Sakli, F. (2009). Physico-chemical characterisation and tensile mechanical properties of Agave americana L. fibres. Journal of the Textile Institute, 100(5), 430-439. https://doi.org/10.1080/00405000701863350

Hulle, A., Kadole, P., \& Katkar, P. (2015). Agave Americana leaf fibers. Fibers, 3(1), 64-75. https://doi.org/10.3390/fib3010064

Mafaesa, M. (2006). The evaluation of conventional retting versus solar baking of agave americana fibres (Master Dissertation, University of Free State, Bloemfontein, South Africa). Recuperado de: http://scholar.ufs.ac.za:8080/xmlui/handle/11660/762?show=full

Msahli, S., Sakli, F., \& Drean, J. Y. (2006). Study of textile potential of fibres extracted from Tunisian agave Americana L. Autex Research Journal, 6(1), 9-13.

Thakur, V. K., Singha, A. S., \& Thakur, M. K. (2013). Ecofriendly Biocomposites from Natural fibers: Mechanical and Weathering study. International Journal of Polymer Analysis and Characterization, 18(1), 64-72. https://doi.org/10.1080/1023666X.2013.747246

Esta obra está bajo una Licencia Creative Commons Attribución-NoCommercial 4.0 International

(cc) BY-NC 